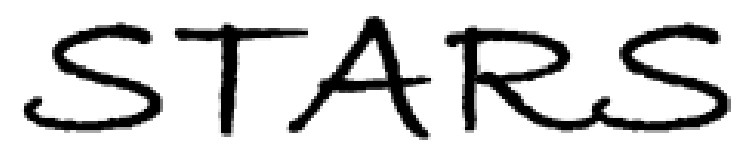

University of Central Florida

STARS

$1-1-2009$

\title{
Tunable two-dimensional plasmon resonances in an InGaAs/InP high electron mobility transistor
}

\author{
H. Saxena \\ University of Central Florida \\ R. E. Peale \\ University of Central Florida \\ W. R. Buchwald
}

Find similar works at: https://stars.library.ucf.edu/facultybib2000

University of Central Florida Libraries http://library.ucf.edu

This Article is brought to you for free and open access by the Faculty Bibliography at STARS. It has been accepted for inclusion in Faculty Bibliography 2000 s by an authorized administrator of STARS. For more information, please contactSTARS@ucf.edu.

\section{Recommended Citation}

Saxena, H.; Peale, R. E.; and Buchwald, W. R., "Tunable two-dimensional plasmon resonances in an InGaAs/InP high electron mobility transistor" (2009). Faculty Bibliography 2000s. 2096.

https://stars.library.ucf.edu/facultybib2000/2096

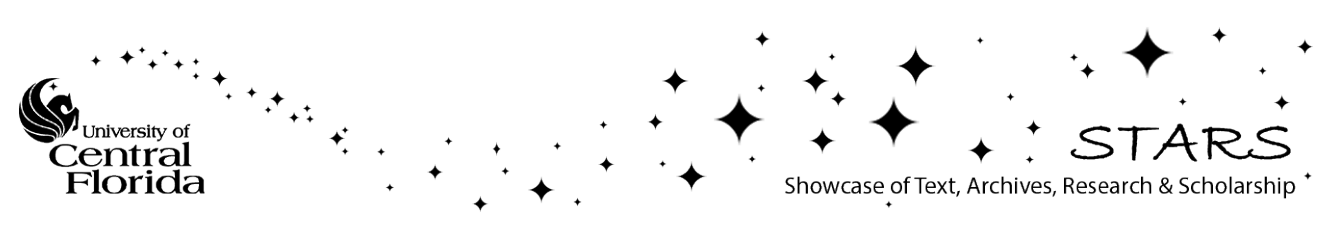




\section{Tunable two-dimensional plasmon resonances in an InGaAs/InP high electron mobility transistor}

Cite as: J. Appl. Phys. 105, 113101 (2009); https://doi.org/10.1063/1.3129319

Submitted: 22 November 2008 . Accepted: 07 April 2009. Published Online: 01 June 2009

H. Saxena, R. E. Peale, and W. R. Buchwald

\section{ARTICLES YOU MAY BE INTERESTED IN}

Temperature dependence of plasmonic terahertz absorption in grating-gate gallium-nitride transistor structures

Applied Physics Letters 96, 042105 (2010); https://doi.org/10.1063/1.3292019

Electrically driven terahertz radiation of 2DEG plasmons in AIGaN/GaN structures at $110 \mathrm{~K}$ temperature

Applied Physics Letters 110, 202101 (2017); https://doi.org/10.1063/1.4983286

Terahertz plasmon amplification using two-dimensional electron-gas layers

Journal of Applied Physics 111, 094501 (2012); https://doi.org/10.1063/1.4709389

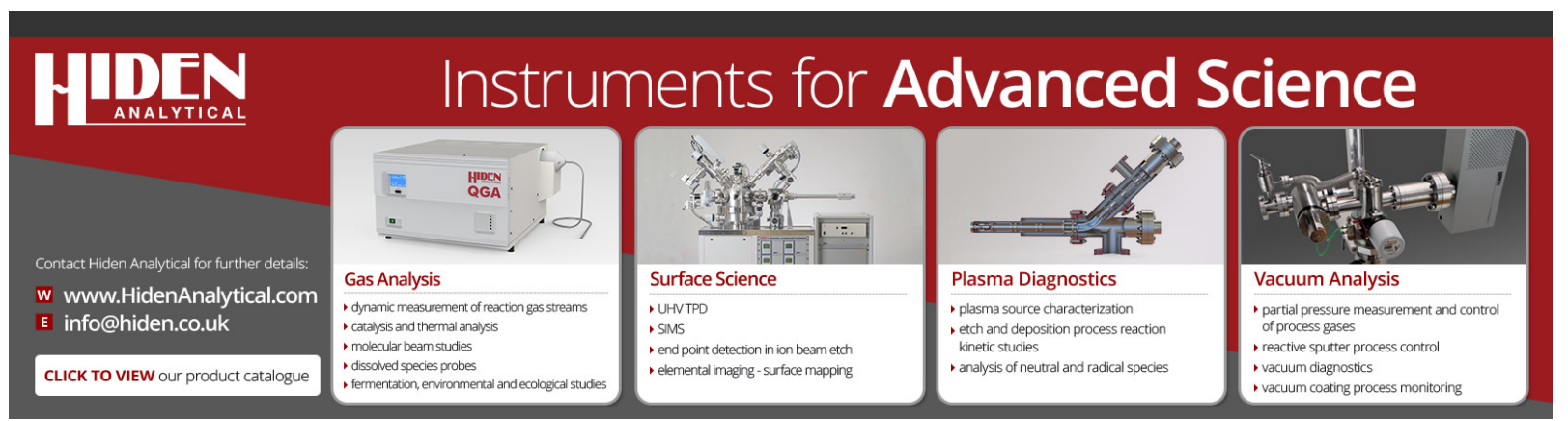

J. Appl. Phys. 105, 113101 (2009); https://doi.org/10.1063/1.3129319

105, 113101

(c) 2009 American Institute of Physics. 


\title{
Tunable two-dimensional plasmon resonances in an InGaAs/lnP high electron mobility transistor
}

\author{
H. Saxena, ${ }^{1}$ R. E. Peale, ${ }^{1, a)}$ and W. R. Buchwald ${ }^{2}$ \\ ${ }_{1}^{1}$ Department of Physics, University of Central Florida, Orlando, Florida 32816, USA \\ ${ }^{2}$ Air Force Research Lab, Sensors Directorate, Hanscom AFB, Massachusetts 01731, USA
}

(Received 22 November 2008; accepted 7 April 2009; published online 1 June 2009)

\begin{abstract}
Voltage-tunable plasmon resonances in the two-dimensional electron gas (2DEG) of a high electron mobility transistor (HEMT) fabricated from the InGaAs/InP materials system are reported. The device was fabricated from a commercial HEMT wafer by depositing source and drain contacts using standard photolithography and a semitransparent gate contact that consisted of a $0.5 \mu \mathrm{m}$ period transmission grating formed by electron-beam lithography. Narrow-band resonant absorption of terahertz radiation was observed in transmission in the frequency range of $10-50 \mathrm{~cm}^{-1}$. The resonance frequency depends on the gate-tuned sheet charge density of the 2DEG. The observed separation of resonance fundamental from its harmonics and their shift with gate bias are compared with theory. (C) 2009 American Institute of Physics. [DOI: 10.1063/1.3129319]
\end{abstract}

\section{INTRODUCTION}

Resonant absorption of terahertz radiation by plasma oscillations in two-dimensional electron gases $(2 \mathrm{DEGs})^{1,2}$ can affect source-drain conductance, a photoresponse that is the basis for proposed frequency-agile terahertz detectors. ${ }^{3}$ Sheet charge density and the characteristic length scale of the gate metallization determine the plasmon resonance frequency. Gate bias control of the sheet charge density allows continuous tuning of the resonance over a wide range.

Figure 1 presents a schematic of the plasmon excitation mechanism in a high-electron mobility transistor (HEMT) by a metallic transmission grating that also serves as the gate contact. The terahertz wave is incident along the surface normal. Figure 1 presents only the electric field component that is polarized perpendicular to the grating stripes. The terahertz wavelength is much larger than the grating period $a$, which in turn much exceeds the distance $d$ between grating and 2DEG. The grating becomes polarized by the terahertz field due to the strongly modulated conductivity normal to the grating stripes. This gives rise to local fields having the period of the grating. In Fig. 1 field lines are drawn schematically to suggest these local fields. (This schematic representation ignores the distortion by the underlying $2 \mathrm{D}$ conducting layer and also by any ultrathin metallization that may be present between the stripes.) These local fields couple to the 2DEG and excite 2D plasmons. The grating period therefore defines the wavevector of the excited plasmon. The resonance frequency is determined from the plasmon wavevector and the plasmon dispersion relation.

A variety of materials systems such as $\mathrm{GaAs} / \mathrm{AlGaAs},{ }^{3}$ InGaP/InGaAs/GaAs, ${ }^{4}$ GaN/AlGaN, ${ }^{2,5}$ and Si (Ref. 1) have been explored, all except the last forming high electron mobility transistors (HEMTs) while the Si system was a metaloxide-semiconductor field-effect transistor (MOSFET). This paper reports terahertz plasma resonances in a HEMT fabricated in the InGaAs/InP system, which features high sheet

${ }^{a)}$ Electronic mail: rep@physics.ucf.edu. charge density and low electron effective mass. Thus, for the same feature size, this material system has potential to realize fundamental plasmon resonances at wavelengths shorter than those previously obtainable in $\mathrm{Si}$ (Ref. 1) or GaAs. ${ }^{3}$

\section{THEORETICAL CONSIDERATIONS}

Calculation of resonance frequencies and terahertz absorption spectra requires (among other material parameters) knowledge of the sheet charge density $n_{s}$ and the temperature dependent relaxation time $\tau$. Both are accessible from electrical characterization of the actual fabricated devices. Measured $I V$ curves determine the source-drain saturation current $I_{\text {sat }}$ as a function of gate bias $V_{\mathrm{g}}$ and temperature, according to 6

$$
I_{\text {sat }}=e n_{s} v_{s} W\left[\sqrt{ }\left(1+\alpha^{2}\right)-\alpha\right] \text {, }
$$

where $v_{s}$ is the saturation velocity, $e$ is the elemental charge, $W$ is the gate width, and

$$
\alpha=\frac{\varepsilon \varepsilon_{o} \nu_{s} L}{q n_{s} \mu d} .
$$

In Eq. (2), $\varepsilon$ is the relative permittivity in the quantum well, $\varepsilon_{0}$ is the permittivity of free space, $\mu$ is the mobility, and $L$ is the gate length (source to drain separation). Also,

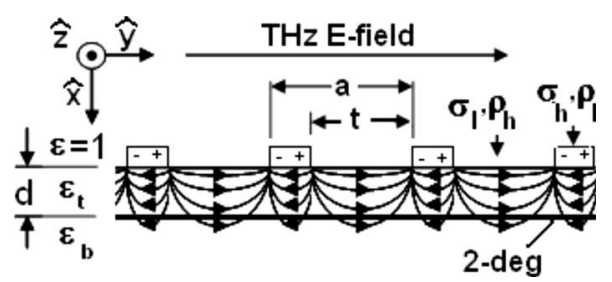

FIG. 1. Schematic of device. The incident terahertz field polarizes the grating, giving rise to local fields that excite plasmons in the 2DEG. 


\begin{tabular}{|c|}
\hline $250 \AA ̊$ In $0.53 \mathrm{Ga} 0.47 \mathrm{As} 4 \times 10^{18} \mathrm{~cm}^{-3} \mathrm{Cap}$ \\
\hline $200 \AA ̊$ In 0.52 Alo.48As Undoped \\
\hline $200 \AA ̊ \operatorname{In} 0.52 \mathrm{Al}_{0.48} \mathrm{As}$ Si doped $4.0 \times 10{ }^{12} \mathrm{~cm}^{-3}$ \\
\hline $45 \AA ̊$ In $0.52 \mathrm{Al}_{0.48}$ As Set Back Undoped \\
\hline $400 \AA ̊$ In $0.53 \mathrm{Ga} 0.47$ As Channel \\
\hline $3000 \AA \operatorname{In}_{0.52} \mathrm{Al}_{0.48}$ As Buffer Undoped \\
\hline Semi-insulating InP substrate \\
\hline
\end{tabular}

FIG. 2. MBE grown epilayer structure used for fabrication of the HEMT.

$$
n_{s}=\varepsilon_{t} \varepsilon_{0} \frac{\left(V_{\mathrm{g}}-V_{t}\right)}{e d}
$$

where $V_{\mathrm{g}}$ is the gate bias, $\varepsilon_{t}$ is the relative permittivity of the material that separates the gate metal from the quantum well, and the threshold voltage is

$$
V_{t}=\phi_{b}-E_{c}-\frac{e n_{d} d}{\varepsilon_{0} \varepsilon_{t}},
$$

where $\phi_{b}$ is the gate Schottky contact barrier height. For metal on III-V compounds we have typically $\phi_{b}=0.7 \mathrm{eV}$. To determine $\varepsilon_{t}$, we note that the permittivity of semiconductor alloy $A_{1-x} B_{x}$ is determined from the permittivities $\varepsilon_{A}$ and $\varepsilon_{B}$ of the end members $A$ and $B$ according to

$$
\varepsilon_{A B}=(1-x) \varepsilon_{A}+x \varepsilon_{B}-x(1-x),
$$

for both InGaAs and InAlAs. ${ }^{7,8}$ The conduction band offset $E_{c}=0.384+0.254 x$ when the In mole fraction $x$ is less than $54 \%$. ${ }^{9}$ The channel in our device (Fig. 2) has $x=0.53$, giving $E_{c}=0.52 \mathrm{eV}$. In Eq. (4) $n_{d}$ is the temperature dependent carrier concentration in the absence of a quantum well. At room temperature, $n_{d}$ is nominally the delta-doping donor concentration.

Combining Eqs. (1)-(4), one sees that measured $I_{\text {sat }}$ versus $V_{\mathrm{g}}$ curves depend on parameters $\mu$ and $n_{d}$, which may be determined from a fit to the data, all other factors considered as known. These $\mu$ and $n_{d}$ values can then be used to determine the 2DEG sheet charge density $n_{s}$ [Eqs. (3) and (4)] and relaxation time $\tau=m^{*} \mu / e$ needed to calculate plasmon resonance frequencies and absorption-line spectra. (Here, $m^{*}$ is the effective electron mass.)

The dispersion relation for plasmons in a $2 \mathrm{DEG}$ is ${ }^{1}$

$$
\omega=\sqrt{\left(\frac{n_{s} e^{2}}{m^{*}}\right)\left\{\frac{g_{p}}{\varepsilon_{o}\left[\varepsilon_{b}+\varepsilon_{t} \operatorname{Coth}\left(g_{p} d\right)\right]}\right\}},
$$

where $\varepsilon_{b}$ is the relative permittivity of the material below the 2DEG and $g_{p}$ is the plasmon wavevector, which can take discrete values $2 \pi p / a$, where $a$ is the grating period. To obtain the highest fundamental plasmon frequencies, short grating periods, high sheet charge density, and a small effective mass are desired. For our $a$ and $d$ values, the hyperbolic cotangent in the denominator of Eq. (6) decreases strongly with $p$ for small $p$, leading to frequencies for the low harmonics that increase more rapidly than the square root of the resonance order.
Equation (6) gives no information concerning the effects of temperature, grating geometry, or material parameters on the strength and spectral shape of the resonant absorption. For this we use the theory of Ref. 10, which treats the excitation of $2 \mathrm{D}$ plasmons by local fields that arise from polarization of the grating by the external terahertz field. Figure 1 presents a schematic of the device geometry. The transmittance $T$ of unpolarized light is

$$
\left(T_{y}+T_{z}\right) / 2 \text {, }
$$

where $T_{y}$ (in Gaussian units) is

$$
T_{y}=\sqrt{\varepsilon_{b}}\left|\frac{2}{1+\sqrt{\varepsilon_{b}}+\Sigma_{y y} \frac{4 \pi}{c}}\right|^{2},
$$

and $T_{z}$ is obtained by substituting $z$ for the $y$ subscript. The complex $y y$-component of the conductivity tensor is

$$
\Sigma_{y y}=\sigma(\omega)+\sigma_{y y}^{g}
$$

while the $z z$-component is given by

$$
\Sigma_{z z}=\sigma(\omega)+\left\langle\sigma_{z z}^{g}\right\rangle
$$

where \langle\rangle indicates a spatial average, and $\sigma(\omega)$ is the conductivity associated with the 2DEG only. The second terms in Eqs. (9) and (10) are components of the grating conductivity tensor and are hence labeled by the superscript $g$. The $\sigma_{y y}{ }^{g}$ is associated with transverse grating currents, which give rise to the grating polarization and local fields that interact with the 2DEG. The $\left\langle\sigma_{z z}{ }^{g}\right\rangle$ is independent of the 2DEG because of the lack of fringing field components polarized in the $z$-direction. All off-diagonal components of $\Sigma$ vanish in the absence of a magnetic field.

The frequency dependent conductivity of the $2 \mathrm{DEG}$ is

$$
\sigma(\omega)=\frac{n_{s} e^{2} \tau}{m^{*}\left(1+\omega^{2} \tau^{2}\right)} .
$$

Following the example of Ref. 10, we take $\tau$ to be the Drude relaxation time determined from the mobility. The last term of Eq. (9) is treated (in the perturbative approximation) as in Ref. 10 in terms of the resistivity tensor, according to which

$$
\sigma_{y y}^{g}=\frac{1}{\langle\rho\rangle}-\sum_{m>0}\left(\frac{\tilde{\rho}(m)}{\langle\rho\rangle}\right)^{2} \frac{F_{m}}{2}
$$

where $\langle\rho\rangle$ is the spatially averaged 2D resistivity of the grating, $\widetilde{\rho}(m)$ is the $m^{\text {th }}$ Fourier coefficient in the cosine expansion of the $y y$-component for the grating resistivity tensor given by

$$
\tilde{\rho}(m)=\frac{2}{\pi m}\left(\rho_{h}-\rho_{l}\right) \sin \frac{\pi t m}{a},
$$

and 


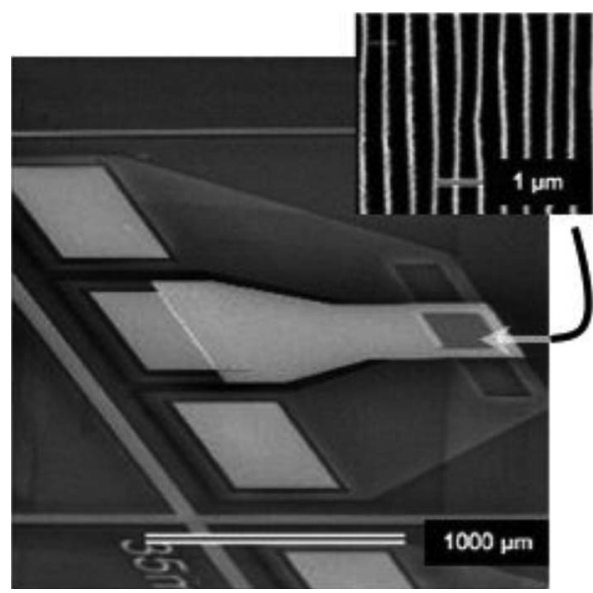

FIG. 3. SEM micrograph of device. The large pads on the left of the image are for wire bonding. The inset is a blowup of the gate region, showing the grating metallization.

$$
\begin{aligned}
F_{p}= & \left(i \omega / 4 \pi g_{p}\right)\left\{1+\varepsilon_{t} \operatorname{Coth}\left(g_{p} d\right)\right. \\
& \left.+\frac{\varepsilon_{b}^{2}\left[1-\operatorname{Coth}^{2}\left(g_{p} d\right)\right]}{\frac{4 \pi i}{\omega} g_{p} \sigma(\omega)+\varepsilon_{b}+\varepsilon_{t} \operatorname{Coth}\left(g_{p} d\right)}\right\} .
\end{aligned}
$$

Following Ref. 10, the sum of Eq. (12) is truncated after $m$ $=10$.

\section{EXPERIMENTAL DETAILS}

The molecular beam epitaxy (MBE) grown epitaxial layer structure for the device of this work is shown in Fig. 2. The $100 \mathrm{~mm}$ diameter wafer is commercial HEMT material purchased from IQE. The $25 \mathrm{~nm}$ thick cap is removed before gate metallization using a 27:1 selective etch, putting the 2DEG in the InGaAs channel at a distance $d=44.5 \mathrm{~nm}$ below the gate.

Figure 3 presents a scanning electron micrograph of the device metallization, which is that of a typical HEMT with a few notable exceptions. To maximize optical throughput at the expense of device switching speed, the gate was made rather large, with length $L=195 \mu \mathrm{m}$ and width $W$ $=250 \mu \mathrm{m}$. Also, the metallization of the entire 3.5 $\times 3.5 \mathrm{~mm}^{2}$ die is designed such that any incident radiation can only pass through the gated area. An interlevel dielectric of $B$-staged bisbenzocyclobutane-based (BCB) polymer was used throughout the fabrication process so that all metal layers could overlap each other to block light transmission outside the gate while maintaining electrical isolation. With the addition of a metal layer around the entire device, the gate acts as an aperture for a detector placed behind the device during spectroscopic characterization. Fabrication was undertaken using standard optical contact lithography and a combination of wet chemical etching for the semiconductor and dry etching for the BCB dielectric. No BCB was left over the gate opening in order to maximize optical throughput. After removal of the InGaAs cap in the gate region, the gate/grating was formed in two steps. A thin Ti layer (7.5 $\mathrm{nm}$ ) was first evaporated over the entire patterned gate area. This thickness is sufficiently less than the terahertz skin depth to allow for transmittance measurements. It was made continuous in order to achieve the most uniform gate control of the 2DEG sheet charge. The gate/grating was then fabricated using e-beam lithography. After spin coating with positive tone PMMA, $30 \mathrm{keV}$ electrons were used to pattern grating stripes with a period of $0.5 \mu \mathrm{m}$. A metal stack of 150 $\AA / 1000 \AA \mathrm{Ti} / \mathrm{Au}$ was then evaporated and lifted, completing the fabrication of the grating gate. The Fig. 3 inset presents a higher magnification image of the gate/grating metallization.

Room temperature Hall measurements were performed on the Fig. 2 structure. In this case, the InGaAs cap was first removed by chemical etching and the material cleaved into a square. Ohmic contacts were added to the four corners of the square with In solder to facilitate the Hall measurements. Temperature dependent transport measurements were performed in a closed cycle cryostat with silicon diode temperature sensor.

For spectroscopic characterization, the device is mounted at the end of the light baffle within the helium cryostat of a $4 \mathrm{~K}$ silicon bolometer (IR laboratories). Since the device is not in direct contact with the $4 \mathrm{~K}$ cold plate, and is further thermally isolated by being mounted on a disk of printed-circuit board material, its temperature is somewhat elevated, probably above $12 \mathrm{~K}$ as deduced below. The opening to the bolometer is carefully screened so that all radiation reaching the bolometer must have passed through the gate region of the device. The sample is mounted in this unconventional way to achieve close coupling of the bolometer to the sample, since diffraction by the subwavelength gate aperture leads to rapid divergence of the beam and low throughput. The bolometer then records the modulated light from a Fourier spectrometer in the $10-50 \mathrm{~cm}^{-1}$ range using a $50 \mu \mathrm{m}$ mylar pellicle beamsplitter and $\mathrm{Hg}$ arc lamp. With a wire-grid on polyethylene polarizer, a strong polarization effect is observed, confirming that the bulk of the recorded signal has passed through the grating gate. When the electric field vector is parallel to the direction of the grating lines, no transmitted signal could be detected. When the electric field vector is rotated to be perpendicular to the grating lines, sufficient signal is transmitted to record a power spectrum.

\section{RESULTS}

Measured $I V$ curves are presented in Figs. 4 and 5. The inset of Fig. 4 presents a schematic of how the device was wired for the $I V$ measurements. Gate voltages $V_{\mathrm{g}}$ and source voltage $V_{\mathrm{s}}$ are referenced to the drain, which is grounded. Figure 4 presents a family of transfer curves $\left(I_{\text {sd }}\right.$ versus $\left.V_{\mathrm{g}}\right)$ indicating proper gate control. At room temperature, $I_{\mathrm{sd}}$ is pinched off at about $V_{\mathrm{g}}=-400 \mathrm{mV}$, while at $22 \mathrm{~K}$ this occurs around $-200 \mathrm{mV}$. Figure 5 presents a family of $I_{\text {sd }}$ versus $V_{\mathrm{s}}$ curves at room temperature and at $22 \mathrm{~K}$ for $V_{\mathrm{g}}$ in the range from 0 to $-0.4 \mathrm{~V}$. At $V_{\mathrm{s}}$ values in the range $100-200 \mathrm{mV}, I_{\mathrm{sd}}$ saturates.

Figure 6 presents the saturation current versus $V_{\mathrm{g}}$ for three temperatures. Here the decreasing $V_{\mathrm{g}}$ required for pinch-off as temperature is decreased is evident. Fits of Eqs. 


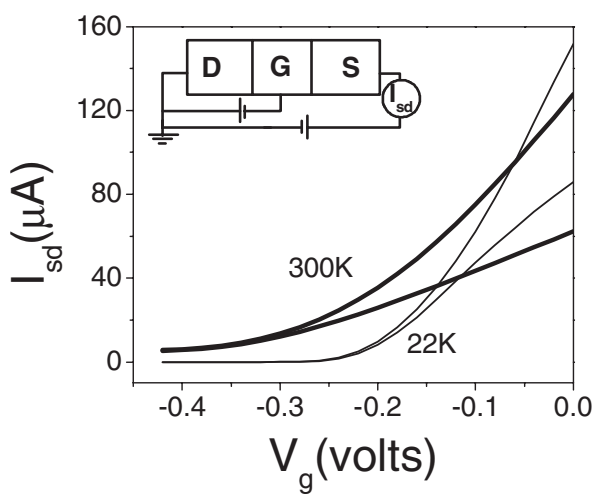

FIG. 4. Transfer curves. Source-drain current as a function of gate bias at various source biases and two different temperatures as indicated. The inset is a wiring diagram for the $I V$ measurements. For each temperature, data was acquired at $V_{\mathrm{s}}=0.1$ (lower curve) and $0.3 \mathrm{~V}$ (upper curve).

(1)-(4) to the data are also plotted, and the fitting parameters $\mu$ and $n_{d}$ are tabulated in the inset for the three temperatures. In these fits, the In mole fraction $x=0.532, v_{s}=1.6$ $\times 10^{5} \mathrm{~m} / \mathrm{s}, \varepsilon_{t}=12.24$, and $\varepsilon_{b}=13.65$. [Permittivities were calculated from Eq. (5) using $\varepsilon_{\mathrm{AlAs}}=10.2, \varepsilon_{\mathrm{InAs}}=14.6$, and $\varepsilon_{\mathrm{GaAs}}=13.1$.] An effective mass $m^{*}=0.043$ is taken for the electrons in the InGaAs channel, ${ }^{11}$ although the actual value may be as much as $50 \%$ higher due to wave-function bleeding into the InAlAs barrier material. ${ }^{12}$ We note that the room temperature zero-bias value of mobility, $\mu=7250 \mathrm{~cm}^{2} / \mathrm{V} \mathrm{s}$ is smaller than both our own Hall value of $11331 \mathrm{~cm}^{2} / \mathrm{V} \mathrm{s}$ and that specified by the manufacture of $10100 \mathrm{~cm}^{2} / \mathrm{V} \mathrm{s}$. Saturation current and Hall measurements are performed for very different biasing conditions, which can easily explain the difference. The $n_{d}$ value of $9.3 \times 10^{11} \mathrm{~cm}^{-2}$ is $\sim 4 \times$ less than the nominal delta-doping level value of $4 \times 10^{12} \mathrm{~cm}^{-2}$.

From the mobility values, relaxation times $0.18,0.44$, and $0.60 \mathrm{ps}$ at 300,22 , and $12 \mathrm{~K}$ are found, respectively. They do not follow the $T^{3 / 2}$ dependence ${ }^{13}$ one expects from temperature dependent phonon scattering, suggesting that temperature-independent impurity, alloy, or interface scattering are important. Thus, the plasmon resonance line width is not expected to decrease continuously with decreasing temperature.

Figure 7 presents plasmon resonance frequencies calculated from Eq. (6) using $a=0.5 \mu \mathrm{m}$. The $p$ values indicate fundamental and harmonics. The $n_{s}$ values at zero gate bias

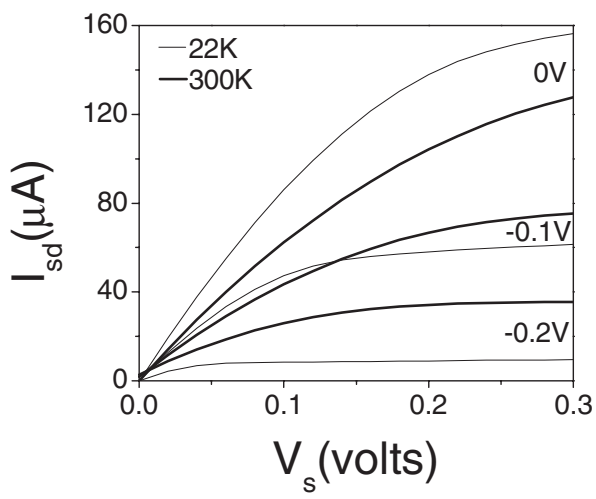

FIG. 5. Source-drain current as a function of source-drain bias at various gate voltages and for two different temperatures.

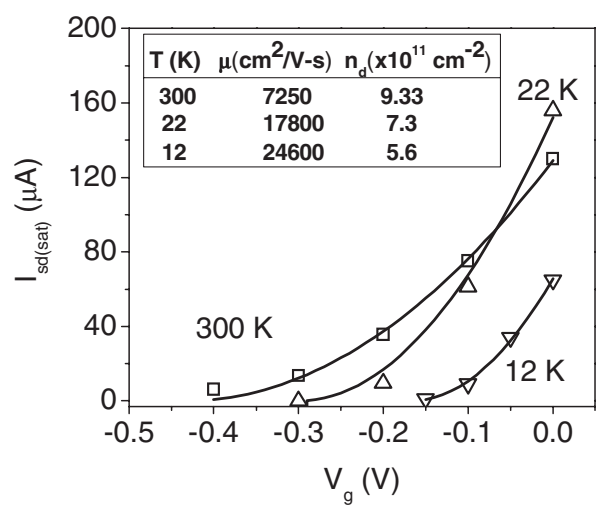

FIG. 6. Curve fits of saturation current as a function of gate bias at various temperatures. The fit parameters $\mu$ and $n_{d}$ are tabulated in the inset.

determined from Eqs. (3) and (4) using $n_{d}$ values from Fig. 6 are indicated by symbols in Fig. 7 for three temperatures. These results indicate a fundamental frequency of $56.3 \mathrm{~cm}^{-1}$ at $22 \mathrm{~K}$ and $V_{\mathrm{g}}=0$. Our room temperature Hall data indicate a sheet charge density of $1.17 \times 10^{12} \mathrm{~cm}^{-2}$, which is $40 \%$ higher than the $6.7 \times 10^{11} \mathrm{~cm}^{-2}$ value obtained from the $I_{\text {sat }}$ curve fitting. For the $I_{\text {sd sat }}$ measurements, the added gate contact might slightly increase the surface depletion.

Figure 8 presents transmission spectra calculated for $T$ $=22 \mathrm{~K}$, where $n_{s}$ values are 4.6, 3.0, and $1.5 \times 10^{11} \mathrm{~cm}^{-2}$ at $V_{\mathrm{g}}=0,-0.1$, and $-0.2 \mathrm{~V}$, respectively. A grating duty cycle $t / a=0.8$ was taken according to Fig. 3. All spectra presented in Fig. 8 have been divided by a calculated spectrum with $t / a=1$, (i.e., with no grating) to divide out the strong baseline slope caused by free carrier absorption. A strong fundamental resonance is observed with weaker higher order resonances also present. All peaks shift to lower wavenumber with negative gate bias. The higher order resonances shift faster, so that the spacing between resonances decreases. The lines also become weaker as $n_{s}$ is depleted.

Figure 9 presents experimental spectra of transmitted intensity for different applied gate voltages with both source and drain grounded. There are strong rapid oscillations due to Fabry-Pérot resonance in the plane-parallel semiconductor substrate. At certain wavenumber positions indicated by arrows, we observe a near cancellation of the oscillations, indicating that the sample is absorbing at these wavenum-

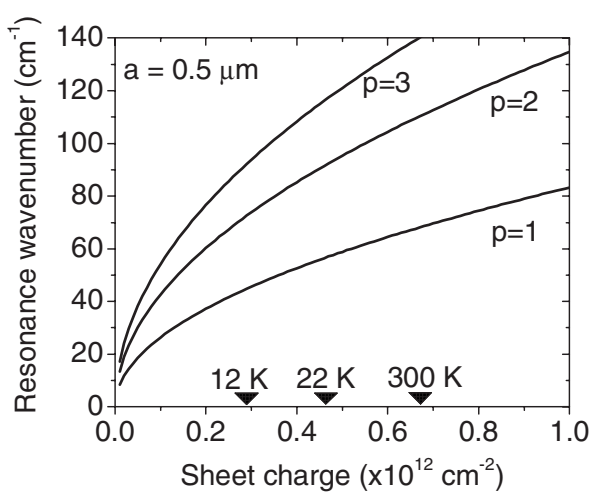

FIG. 7. Calculated plasmon resonance wavenumbers as function of sheet charge density. The symbols on the abscissa indicate values of $n_{s}$ for $V_{\mathrm{g}}$ $=0$ determined from experimental $I V$ curves at the three temperatures indicated. 


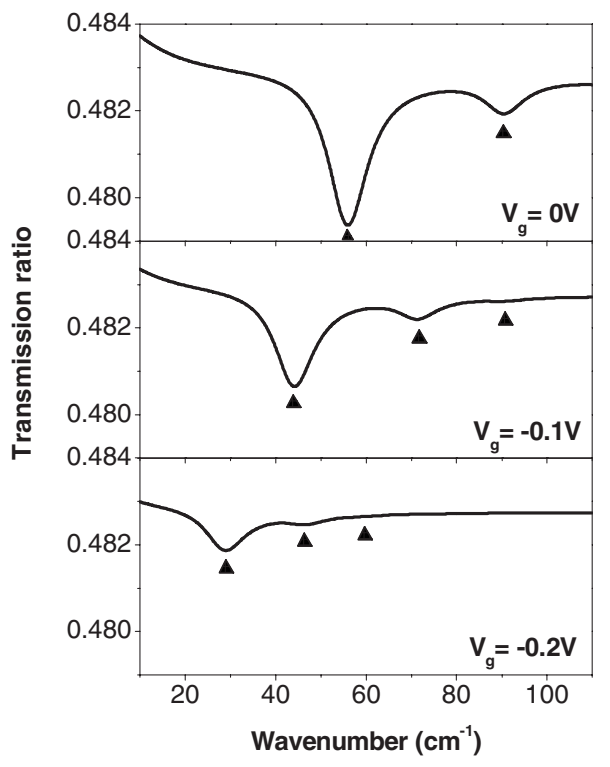

FIG. 8. Calculated transmittance spectra. Each spectrum is divided by a spectrum with zero grating stripe width to eliminate baseline effects due to free carrier absorption. Remaining are absorption lines due to plasmons. Spectra are presented for three different values of gate voltage at a sample temperature of $22 \mathrm{~K}$.

bers. (That some of the signal is negative and that some absorption peaks appear with the wrong sign is attributed to the extremely weak throughput leading to poor phase correction of the single-sided interferogram.) For zero gate bias, two absorption resonances are observed, and by $V_{\mathrm{g}}=$ $-0.2 \mathrm{~V}$, a third absorption feature has emerged into the high end of the spectral range of the experiment. As negative gate voltage is applied, all features shift to lower wavenumbers and converge, in qualitative agreement with the calculated spectra (Fig. 8). Also in agreement with the calculated spectra is the tendency for the lowest resonance to be stronger than the higher ones. Hence we attribute these features to the plasmon resonances.

Figure 10 compares the predicted resonance frequencies

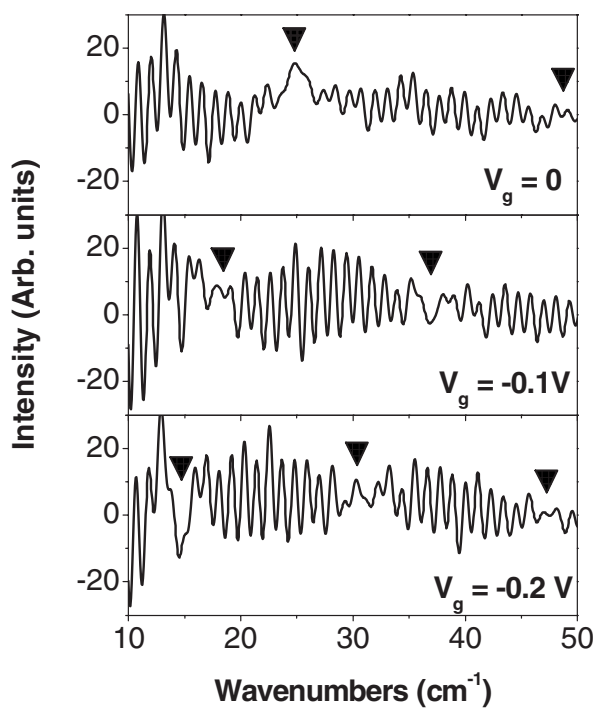

FIG. 9. Measured spectrum of intensity transmitted through gate region of HEMT. Plasmon absorptions (arrows) appear as the extinction of substrate Fabry-Pérot oscillations.

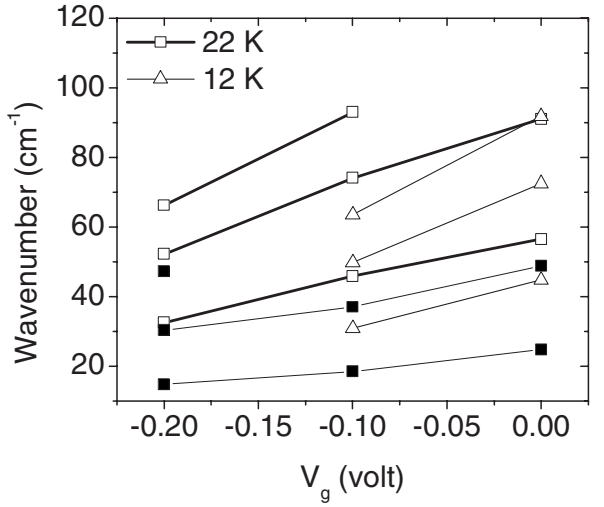

FIG. 10. Comparison of resonance wavenumber as a function of gate bias calculated for two different temperatures (open symbols) with experimental data (solid symbols).

[Eq. (6), open symbols] versus $V_{\mathrm{g}}$ for two different temperatures and for $p=1,2$, and 3 harmonics with the experimental observations (solid symbols). We note that the observed resonances occur at lower wavenumbers than predicted by a factor of up to $\sim 3$. At $12 \mathrm{~K}$, the sheet charge density is already depleted at $-0.2 \mathrm{~V}$ gate bias, according to our transport measurements, but our experiment still gives evidence of plasmons. For this reason we believe that the sample temperature exceeded $12 \mathrm{~K}$ during the transmission measurements.

\section{DISCUSSION AND SUMMARY}

We note two types of discrepancies between the calculated and observed resonances. These are in the ratios of the higher resonance frequencies to the fundamental frequency and in the absolute frequency values. According to Eq. (6), the ratios are affected only by the value of $d / a$ and not by the value of $n_{s} / \mathrm{m}^{*}$. [Here, we consider the uncertainties in the permittivities to be small, because all the end member values used in Eq. (5) are similar.]

Calculated ratios $\omega_{p}: \omega_{1}$ from Eq. (6) with the nominal $d / a$ value are 1.6, 2.1, 2.4, and 2.7 for $p=2,3,4,5$, respectively. The observed ratios (Fig. 9) are approximately $2.0 \pm 0.1$ and 3.2, where the uncertainty quoted is due to scatter when there is more than one data point. These observed ratios are significantly higher than the calculated ones for the same mode numbering.

If one supposes that only odd modes have been observed, as was the case in Refs. 3 and 14, then the observed ratio 2.0 agrees better with the calculated value for $\omega_{3} / \omega_{1}$, but then the observed ratio 3.2 is still significantly higher than the calculated $\omega_{5} / \omega_{1}$. Besides failing to give adequate agreement in the ratios, exclusion of evenly numbered modes is unphysical in the present case. In Refs. 3 and 14, the grating duty cycle was 50\%, and in that case the even-order terms vanish from the Fourier expansion of the local fields, so that even harmonics are not excited by them. The disappearance of even modes for a $50 \%$ duty grating was clearly demonstrated by calculation in Ref. 15. In the present work, the grating duty was far from $50 \%$ (Fig. 3), and even modes are expected.

Closer agreement between calculated and observed ratios is achieved by supposing a smaller $d / a$ value. For suf- 
ficiently small $d / a$, the ratios increase linearly in $p$ since then the Coth term in Eq. (6) goes as $1 / p$. This would give ratios of 2 and 3 in close agreement with observations. Values of $d / a$ that achieve this are below $\sim 0.01$, while the nominal value was 0.089 . Manufacturer-specified layer thicknesses (Fig. 2) should be accurate, and selective etching and step profiling support the nominal $d$ value $44.5 \mathrm{~nm}$. The $a$ value might be replaced by the gate-aperture dimension $L$ as the characteristic length that determines the plasmon wavevector, and since $L$ exceeds $a$ by 390 -fold, such replacement is beyond that necessary to achieve ratios going as $p$. However, this replacement causes serious problems for the absolute frequencies, as explained next.

The second type of discrepancy is that measured resonances fall below the positions calculated using nominal parameter values. With the nominal zero-bias $n_{s} / m^{*}$ value, smaller calculated frequencies can be achieved by reducing $d$ or increasing $a$, (which also improves the ratios). As indicated above, replacing $a$ by $L$ puts the fundamental a factor $\sim 250$ below the lowest observed resonance, so that the observed resonances would have to correspond to high orders, leading again to ratio problems. This might be remedied by a simultaneous $250^{2}$-fold increase in $n_{s} / m^{*}$, but such seems unreasonable, as argued below. Instead, taking again the nominal $a$ and $n_{s} / m^{*}$ values, fair agreement for both absolute frequencies and their ratios is achieved by reducing $d$ to $\sim 5 \mathrm{~nm}$. Although it seems unreasonable to reduce the gate2DEG distance so much, we note that this is approximately the thickness of the undoped InAlAs setback above the 2DEG.

We next consider the probable uncertainty in $n_{s} / \mathrm{m}^{*}$. We have noted that the $m^{*}$ value could be $50 \%$ higher than the nominal value due to wave function penetration into the barrier material. $I V$ measurement gave $n_{s}$ values using simplified equations that nevertheless should give a better than order-of-magnitude estimate of $n_{s}$. Independent Hall measurement gave a $40 \%$ higher $n_{s}$, but the very different geometries of the HEMT and the Hall sample make this value of questionable relevance. Capacitance versus voltage measurements might have been used to estimate $n_{s}$, but this technique was not used here because of ambiguities due to trapping centers and the extent of dopant activation. All these considerations suggest that the nominal $n_{s} / \mathrm{m}^{*}$ value is known with better than order-of-magnitude accuracy.

It may be that the heavy doping of nearly half of the 44.5 $\mathrm{nm}$ of InAlAs layer has a significant effect unaccounted for by the theory, which was developed for silicon MOSFETs where the layer separating the gate from $2 \mathrm{DEG}$ is $\mathrm{SiO}_{2}$. Perhaps the doping, which ends $\sim 5 \mathrm{~nm}$ above the 2DEG, gives rise to an effective value for $d$ of that magnitude. The modification of the Ref. 10 theory to account for the presence and effects of unscreened ionized donor impurities on the fields and charge distribution of the 2DEG is beyond the scope of this paper, and we leave it as a question for theoreticians.

In summary, detection of 2D plasma oscillations in the electron gas of an InGaAs/InP HEMT device has been reported. This device utilizes grating coupling of incident radiation to plasmon modes in the 2DEG. Such a device might find application as a voltage-tunable band-blocking filter. Alternatively, potential changes in device conductance caused by resonant absorption might be used for tunable terahertz detection.

\section{ACKNOWLEDGMENTS}

The authors of this work would like to acknowledge funding for this work from the Air Force Office of Scientific Research Tasks under Grant Nos. 06SN05COR and 92SN06COR. R.E.P. and H.S. acknowledge AFRL Contract No. FA871807C0036.

${ }^{1}$ S. J. Allen, D. C. Tsui, and R. A. Logan, Phys. Rev. Lett. 38, 980 (1977). ${ }^{2}$ W. Knap, Y. Deng, S. Rumyantsev, and M. S. Shur, Appl. Phys. Lett. 81, 4637 (2002).

${ }^{3}$ X. G. Peralta, S. J. Allen, M. C. Wanke, N. E. Harff, J. A. Simmons, M. P. Lilly, J. L. Reno, P. J. Burke, and J. P. Eisenstein, Appl. Phys. Lett. 81, 1627 (2002).

${ }^{4}$ T. Otsuji, M. Hanabe, and O. Ogawara, Appl. Phys. Lett. 85, 2119 (2004).

${ }^{5}$ W. Knap, V. Kachorovskii, Y. Deng, S. Rumyantsev, J.-Q. Lü, R. Gaska, M. S. Shur, G. Simin, X. Hu, M. Asif Khan, C. A. Saylor, and L. C. Brunel, Appl. Phys. Lett. 91, 9346 (2002).

${ }^{6}$ A. A. Grinberg and M. Shur, J. Appl. Phys. 65, 2116 (1989).

${ }^{7}$ M. Littlejohn, K. Kim, and H. Tyan, in Properties of Lattice-Matched and Strained Indium Gallium Arsenide, EMIS Data Reviews Series Vol. 8, edited by P. Bhattacharya (IEEE, New York, 1993).

${ }^{8}$ E. F. Shubert, Doping in III-V Semiconductors (Cambridge University Press, Cambridge, UK, 1993).

${ }^{9}$ J.-H. Huang, T. Y. Chang, and B. Lalevic, Appl. Phys. Lett. 60, 733 (1992).

${ }^{10}$ L. Zheng, W. L. Schaich, and A. H. MacDonald, Phys. Rev. B 41, 8493 (1990).

${ }^{11}$ L. A. Cury, J. Beerens, and J. P. Praseuth, Appl. Phys. Lett. 63, 1804 (1993).

${ }^{12}$ Y. T. Dai, Y. F. Chen, and I. Lo, Phys. Rev. B 55, 5235 (1997).

${ }^{13}$ C. Kittel, Introduction to Solid State Physics, 2nd ed. (Wiley, New York, 1956), p. 352.

${ }^{14}$ E. A. Shaner, M. Lee, M. C. Wanke, A. D. Grine, J. L. Reno, and S. J. Allen, Appl. Phys. Lett. 87, 193507 (2005).

${ }^{15}$ W. R. Buchwald, H. Saxena, and R. E. Peale, Proc. SPIE 6678, 32 (2007). 